\title{
Study of Compton scattering signals in single-sided imaging applications using Monte Carlo methods
}

\author{
L.M.N. Tavora ${ }^{a, b, *}$, W.B. Gilboy ${ }^{c}$ \\ a ESTG, Inst. Politécnico de Leiria, Morro do Lena, 2411-901 Leiria, Portugal \\ ${ }^{\mathrm{b}}$ Centro de Instrumentação, Departamento de Física, Universidade de Coimbra, 3004516 Coimbra, Portugal \\ ${ }^{\mathrm{c}}$ Department of Physics, University of Surrey, Guildford Surrey, GU2 7XH, UK
}

\begin{abstract}
Compton scatter imaging (CSI) is well establish one-sided imaging approach, where the image is formed using photons that are singly scattered in a region of the object. However, the measured data includes also photons that are multiply scattered and is also affected by the fact that incoming and outgoing beams are attenuated. In this paper, the EGS4 code system is used to gain some insight into the physics that constrains CSI. The yields of singly and multiply scattered photons, obtained with monochromatic and X-ray tube inspecting beams, were assessed for a variety of media and depths.
\end{abstract}

(c) 2003 Elsevier B.V. All rights reserved.

\section{Introduction}

The use of ionising radiations in imaging applications is nowadays a well-established technique in medical, industrial and security applications $[1,2]$. Techniques like conventional radiography or computed tomography specifically rely on the fact that photon absorption is highly dependent on the density and atomic number of the material under consideration. These imaging techniques are limited if access to both sides of an object is restricted, or when the thickness is such that very few pho-

\footnotetext{
* Corresponding author. Address: Centro de Instrumentacão, Departamento de Física, Universidade de Coimbra, 3004516 Coimbra, Portugal. Tel.: +351-239-410667; fax: +351239-829158.

E-mail address: 1mtavora@saturno.fis.uc.pt (L.M.N. Tavora).
}

tons are transmitted through it. In such cases, an alternative approach is to use a single-sided imaging method in which source and detector are placed on the same side of the object.

One such method is Compton scatter imaging (CSI) [3] where an image is formed by exploiting those photons that are singly scattered in the accessible regions of the object. Although this method is well suited for material discrimination, some difficulties may arise due to the fact that the measured data includes additional photons that are multiply scattered, and also because incoming and outgoing beams are attenuated in the sample.

The use of Monte Carlo methods to simulate the transport of ionising radiation in matter is acknowledged as a powerful and versatile technique [4], and the EGS4 code system [5] is one of the packages that can be used to model coupled electron/photon interactions in matter. Originally 
developed for high energy physics applications, this tool can nowadays be used down to energies of the order of a few $\mathrm{keV}$, offering the possibility of defining arbitrary geometries and score a wide range of output tallies.

This communication describes the results obtained with the EGS4 Monte Carlo code in the simulation of Compton scattering experiments. The yields of singly scattered photons and ratios of single-to-multiple scattered radiation are presented for different inspecting beams, penetration depths and media.

\section{Compton scattering imaging}

Upon interacting with matter, photons may experience a Compton scattering collision. This can be described as a billiard-ball type of collision between an incident photon and an electron of the target atom. The probability of a photon undergoing a single collision that results in a scattering angle $\theta$ is given, to a good approximation, by the Klein-Nishina differential cross section [6]. This distribution is fairly isotropic for photon energies of a few tens of $\mathrm{keV}$, but strongly peaks in the forward direction as the photon energy increases. Therefore, higher energy photons are more likely to be scattered in the forward direction.

The basic geometry for a CSI experiment, in which the measured signal is composed of photons that are backscattered at a depth $d$ in the object under examination, is presented in Fig. 1. In this kind of configuration both the photon source and the detector are placed on the same side of the sample.

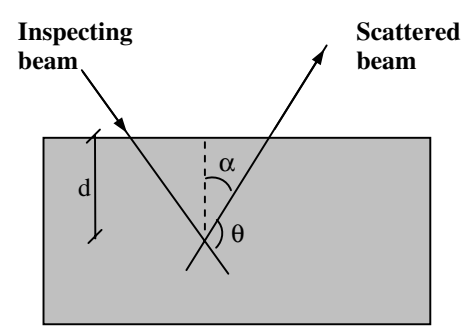

Fig. 1. Configuration for Compton backscatter imaging.

\subsection{Arrangement optimisation}

The first question that arises concerns the relative positions of the radiation source and detector with respect to the sample. In other words, for a given scattering angle $\theta$, how should the system be arranged in order to minimise the attenuation of the incident and outgoing beams? From Fig. 1, the problem can be mathematically stated in the following terms: for a certain depth $d$, and given the quantity

$$
\begin{aligned}
T_{\mathrm{r}} & =T_{\mathrm{r}}^{\text {in }} \times T_{\mathrm{r}}^{\text {out }} \\
& =\exp \left[-\mu_{1} d / \cos (\theta-\alpha)\right] \times \exp \left[-\mu_{2} d / \cos (\alpha)\right],
\end{aligned}
$$

where $\mu_{1}$ and $\mu_{2}$ are, respectively, the linear attenuation coefficients for the inspecting and scattered beams and $T_{\mathrm{r}}^{\text {in }}, T_{\mathrm{r}}^{\text {out }}$ are the transmission factors for the incident and scattered beams, what value of $\alpha$ maximises $T_{\mathrm{r}}$ ?

The maximisation of $T_{\mathrm{r}}$ can only be carried out numerically, but that turns out to be a rather straightforward process. Indeed, the equation $\mathrm{d}\left(T_{\mathrm{r}}\right) / \mathrm{d} \alpha=0$ has been solved using Newton's method [7] in a simple worksheet, and the "optimum" value of $\alpha$ was determined for different materials at incident energies of 150 and $300 \mathrm{keV}$ and $\theta=150^{\circ}$. Although the "most favourable angle" $\alpha$ is independent of the depth under inspection $d$, the factor $T_{\mathrm{r}}$ is not. The results obtained, using photon attenuation data from XCOM [8] are presented in Table 1 for some materials of special interest. These data make it clear that, as the scattered beam is more strongly attenuated than the incoming beam, the system

Table 1

Values of $\alpha$ that minimise beam attenuation in different samples (representing common building materials and others), for beams with different energies

\begin{tabular}{llll}
\hline Material & $\begin{array}{l}\text { Density } \\
\left(\mathrm{g} / \mathrm{cm}^{3}\right)\end{array}$ & \multicolumn{2}{l}{ Optimum angle (deg.) } \\
\cline { 4 - 4 } & 7.93 & 10.4 & $300 \mathrm{keV}$ \\
\hline Steel & 1.76 & 14.1 & 13.8 \\
RDX $\left(\mathrm{C}_{3} \mathrm{H}_{6} \mathrm{~N}_{6} \mathrm{O}_{6}\right)$ & 2 & 13.7 & 13.1 \\
Clay & 0.6 & 14.1 & 13.3 \\
Wood & 0.002 & 15.1 & 13.4 \\
Foam $\left(\mathrm{C}_{8} \mathrm{H}_{8}\right)$ & & & \\
\hline
\end{tabular}


should be positioned with an angle $\alpha$ of the order of only a few degrees.

\subsection{Dependence of the scattering yield on the} photon energy

The dependence of the backscattering yield $(Y)$ on the energy of incident beam can be roughly estimated from

$Y=T_{\mathrm{r}}^{\text {in }} \times N_{\text {ele }}\left(\frac{\mathrm{d} \sigma}{\mathrm{d} \Omega}\right) \times T_{\mathrm{r}}^{\text {out }}$,

where $N_{\text {ele }}$ is electron density of the medium, $T_{\mathrm{r}}^{\text {in }}$, $T_{\mathrm{r}}^{\text {out }}$ represent the transmission fractions of incident/scattered beams, and $\left(\frac{\mathrm{d} \sigma}{\mathrm{d} \Omega}\right)_{\theta}$ is the Compton differential cross section.

As the energy of the incident beam increases, the same happens with the energy of the scattered radiation, thus increasing $T_{\mathrm{r}}^{\text {in }}$ and $T_{\mathrm{r}}^{\text {out }}$. However, since photons increasingly scatter in the forward direction as their energy increases, a compromise between the quantities in (2) should result in an "optimum" energy where $Y$ is maximum. The factor $Y$ has been calculated for different materials, at a depth of $1 \mathrm{~cm}$ and $\theta=150^{\circ}$, and the results obtained are presented in Fig. 2.

\section{Simulated Compton backscattering yields}

The yield of singly scattered photons has been determined with the EGS4 code using the basic set-up presented in Fig. 1, with $\alpha=0$. The materials considered are those listed in Table 1, with inspecting beams of different energies probing several average target depths.

In the first set of studies, EGS4 was used to determine singly scattered photon yields for an average inspection depth of $1 \mathrm{~cm}$. The detector was assumed to be at a distance of $30 \mathrm{~cm}$ from the sample surface with a $1-\mathrm{cm}$-collimator, in a geometry in which source and detector beams were collimated to focus a voxel of $1 \mathrm{~cm}^{3}$. The results obtained are presented in Fig. 4, showing a very good agreement with curves presented in Fig. 3.

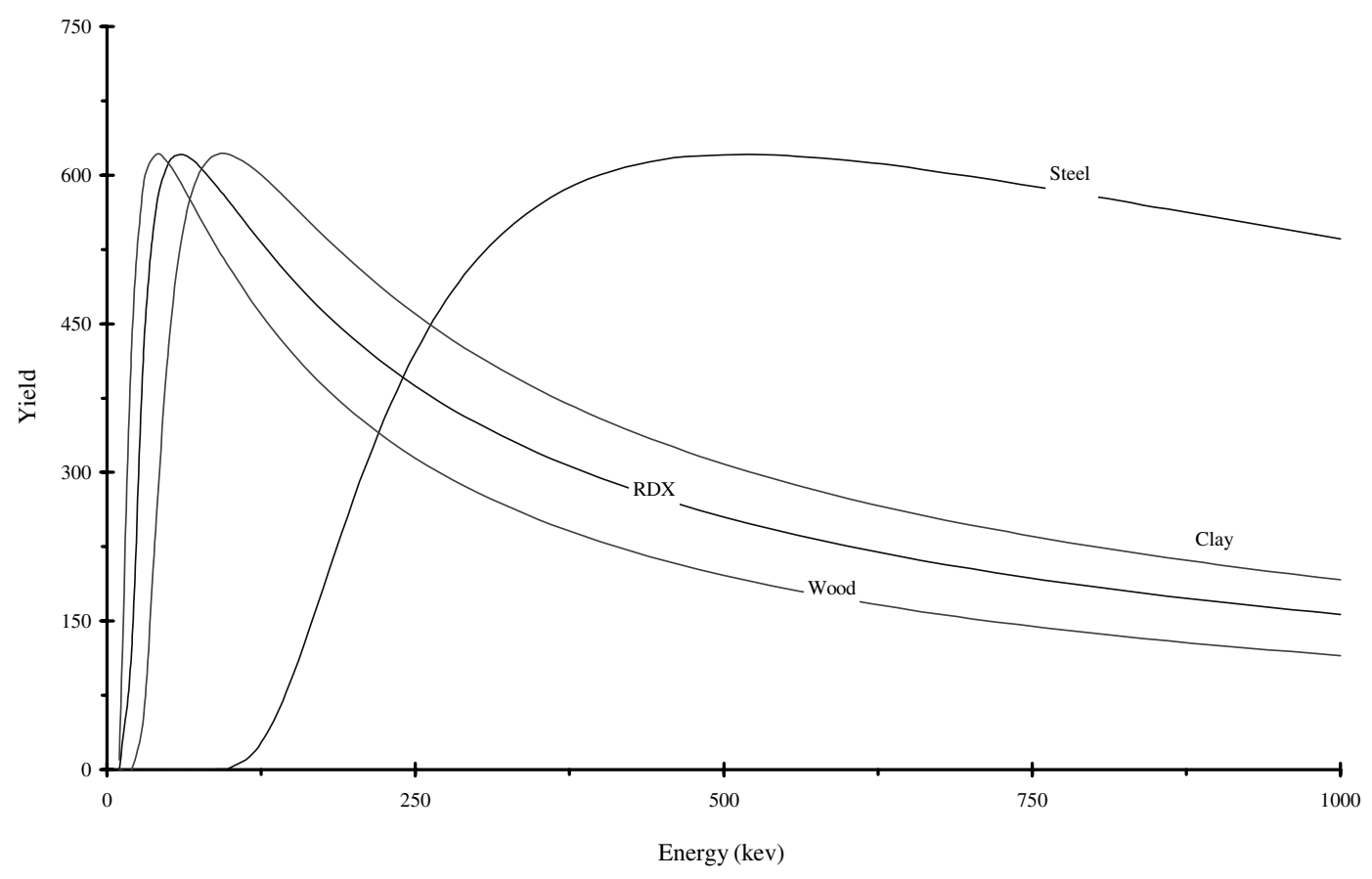

Fig. 2. Dependence of $Y$ (Eq. (2)) on the beam energy for different materials, at a depth of $1 \mathrm{~cm}$, calculated using the XCOM database. All sets were normalised to the same maximum value. 


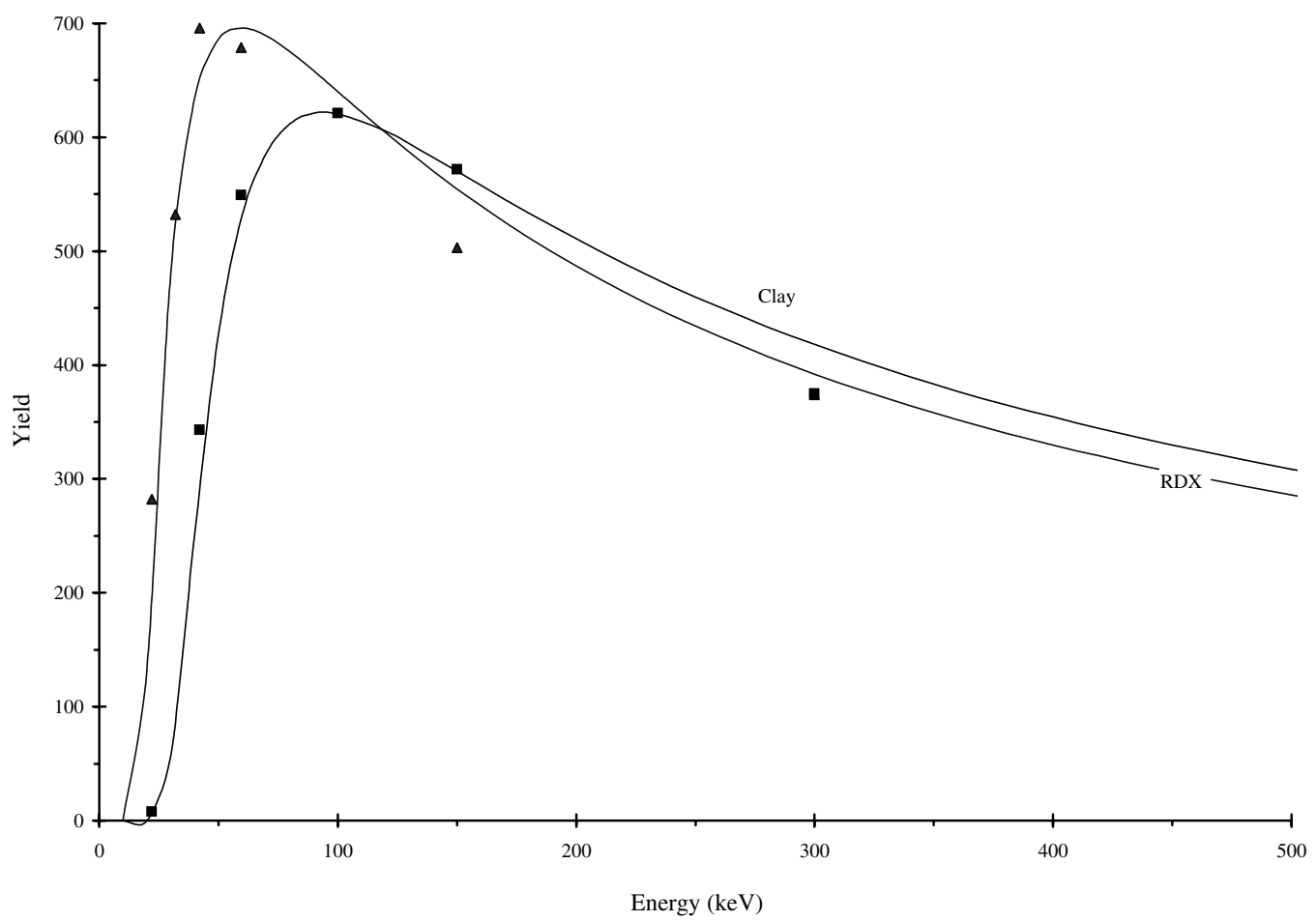

Fig. 3. Single scattering yields versus beam energy. Theoretical estimations and corresponding Monte Carlo data (triangles - RDX; squares - clay) have been normalised to the same maximum value.

The dependence of the scattering yield on the mean depth being probed has also been determined for the same arrangement, and the results are presented in Fig. 4 for different photon energies. These data demonstrate that, as a direct consequence of incident and outgoing beams being more attenuated, going deeper into the sample results in a reduction of the scattering signals. It was also observed that scattered photon spectra from denser materials and deeper locations have higher multiple scattering contributions.

Tungsten target X-ray tube spectra, generated using EGS4/GOS [9], have also been considered in this study as photon sources. Scattered photon spectra, obtained using a $300 \mathrm{kVp}$ inspecting beam and for $d=1 \mathrm{~cm}$, are presented in Fig. 5(a) and (b) for RDX and steel, showing an increased fraction of multiply scattered photons from higher $Z$ materials. The number of singly scattered X-ray photons has been logged at different depths and the results are presented in Fig. 6, for a tube operated at $150 \mathrm{kVp}$.

\section{Conclusions}

EGS4 has been used to study the yield of single and multiple scattered photons in simulations of Compton backscatter imaging experiments, for different materials and various beam energies. Firstly, the arrangement of source and detector has been analysed leading to the practical suggestion that, in order to reduce the overall attenuation of the signals, the outgoing radiation should emerge from the sample along a path closely perpendicular to the sample surface. The incident photon energies that maximise single scattering yields have also been determined for different materials, showing good agreement with some rough theoretical estimates. 

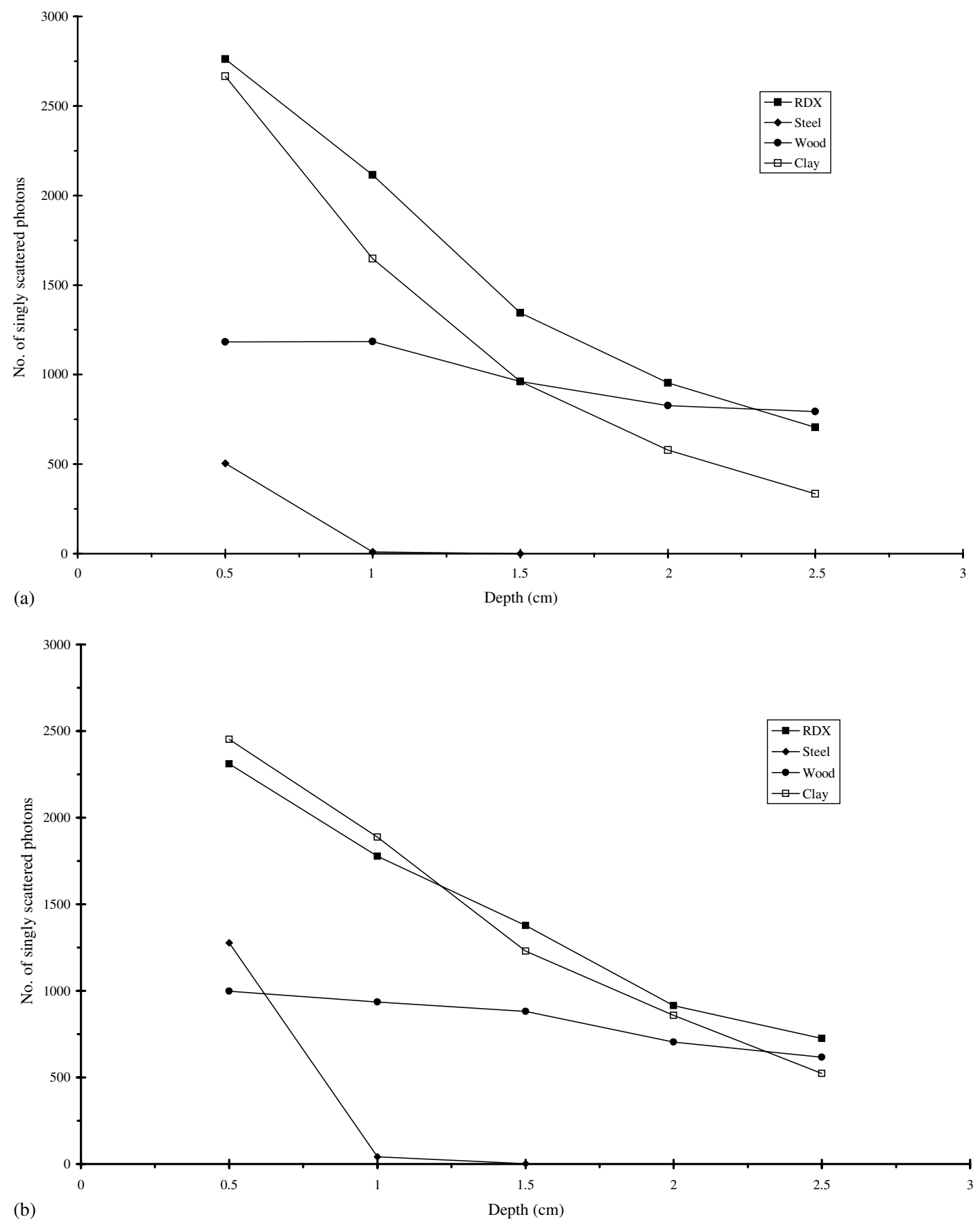

Fig. 4. Single scattering yields versus depth for (a) $60 \mathrm{keV}$ and (b) $100 \mathrm{keV}$ incident beams.

It has also been shown that, due to the increased attenuation and multiple scattering, yields and image contrast (single/multiple scatter ratio) are reduced when deeper regions are being probed. 

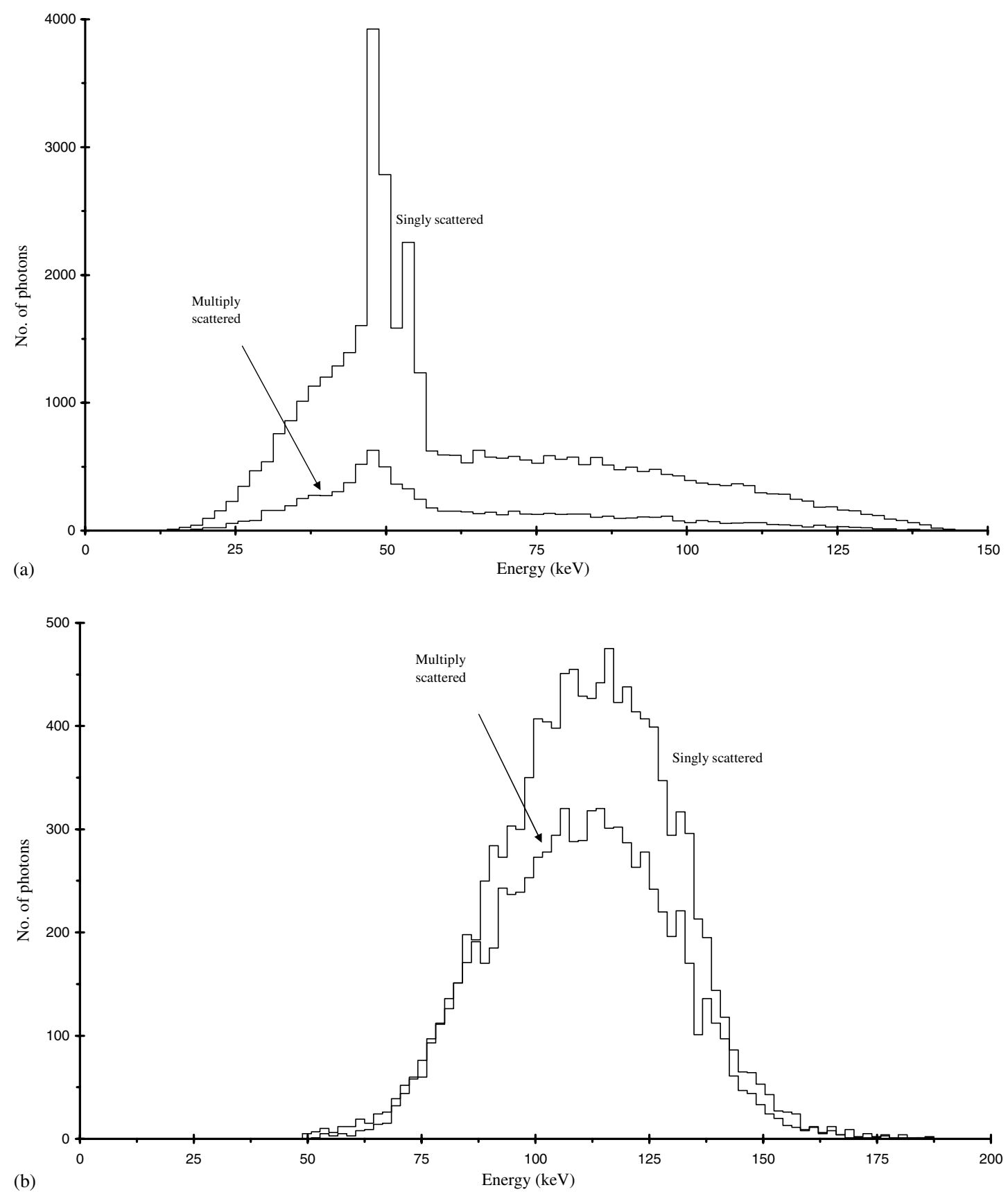

Fig. 5. Scattered photon energy spectra from (a) RDX and (b) steel.

Accordingly, this effect is more penalising for materials of higher density and higher atomic numbers, which may result in situations where the single scattering signals emerging from a given depth in materials with lower electron densities exceed those from denser samples. 


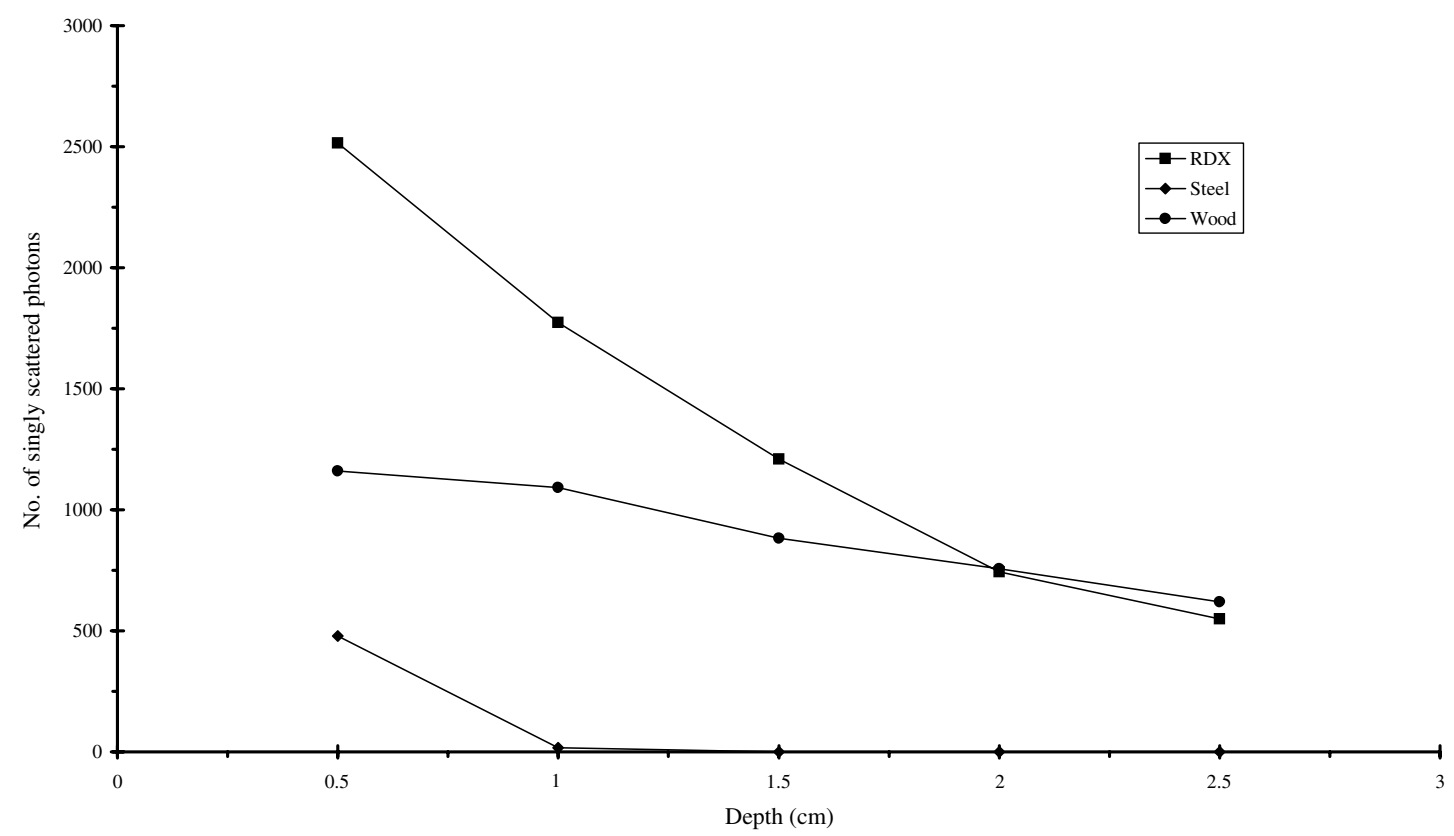

Fig. 6. Yield versus depth using a $150 \mathrm{kVp} \mathrm{W}$-target X-ray tube spectra as the interrogating beam.

\section{Acknowledgements}

This work was carried out in the Departamento de Física da Universidade de Coimbra (Research Unit No. 217/94). L.M.N.T. acknowledges financial support from Fundação para a Ciência e Tecnologia (Portugal) through project CERN/ FIS/43787/2001.

\section{References}

[1] S. Webb (Ed.), The Physics of Medical Imaging, Institute of Physics, Bristol, 1988.
[2] W.B. Gilboy, J. Foster, in: R.S. Sharpe (Ed.), Research Techniques in Nondestructive Testing, Vol. VI, Academic Press, 1982.

[3] G. Harding, Radiat. Phys. Chem. 50 (1997) 91.

[4] T.M. Jenkins, W.R. Nelson, A. Rindi, Monte Carlo Transport of Electrons and Photons, Plenum, 1988.

[5] W.R. Nelson, H. Hirayama, D.W.O. Rogers, Stanford Linear Accelerator Report SLAC-265, 1985.

[6] G.F. Knoll, Radiation Detection and Measurement, 3rd Ed., John Wiley, 2000.

[7] W.H. Press, S.A. Teukolsk, W.T. Vetterling, B.P. Flannery, Numerical Recipes in C - The Art of Scientific Computing, 2nd Ed., Cambridge University, 1992.

[8] M.J. Berger, J.H. Hubbell, National Bureau of Standards Report 87-3757, 1987.

[9] L.M.N. Távora, E.J. Morton, Nucl. Instr. and Meth. B 143 (1998) 253. 